Research, Society and Development, v. 9, n. 11, e50791110081, 2020

(CC BY 4.0) | ISSN 2525-3409 | DOI: http://dx.doi.org/10.33448/rsd-v9i11.10081

\title{
Prospecção tecnológica de internet das coisas no contexto dos hospitais inteligentes
}

Technological prospection of internet of things in the context of smart hospitals

Prospección tecnológica de internet de cosas en el contexto de hospitales inteligentes

Recebido: 10/11/2020 | Revisado: 15/11/2020 | Aceito: 18/11/2020 | Publicado: 24/11/2020

Felipe Roberto Eloi Moura

ORCID: https://orcid.org/0000-0002-7509-1942

Universidade Federal de Alagoas, Brasil

E-mail: felipemoura.al@gmail.com

Guilherme Benjamin Brandão Pitta

ORCID: https://orcid.org/0000-0003-2790-2015

Universidade Federal de Alagoas, Brasil

E-mail: guilhermebbpitta@gmail.com

Josealdo Tonholo

ORCID: https://orcid.org/0000-0002-4015-9564

Universidade Federal de Alagoas, Brasil

E-mail: tonholo@gmail.com

\section{Resumo}

Ancorado no paradigma da internet das coisas (IoT), os Hospitais Inteligentes têm surgido como uma proposta inovadora de hospitais, onde as funções de diagnósticos, tratamento, gestão e decisão estão integradas. Diante dos grandes problemas que hospitais no mundo todo tem enfrentado, a necessidade de um ambiente inteligente dentro do contexto hospitalar tem sido urgente, e os benefícios trazidos pela IoT para este setor são inúmeros. Entendendo a importância deste tema, este trabalho se propôs a realizar um estudo de prospecção tecnológica em base de patentes e artigos científicos, a fim de investigar tecnologias baseadas no paradigma da IoT com aplicação em ambientes hospitalares. Assim, foram realizadas buscas nas bases Questel-Orbit, para documentos de patentes, e Scopus, para artigos científicos. Através das análises patentárias e bibliométricas foi possível construir um mapeamento das tecnologias e pesquisas científicas sobre IoT com aplicação em hospitais, dentro do corte temporal de 2009 a 2020. Os resultados apontaram para um crescimento exponencial de depósitos de patentes e publicações de artigos sobre este tema. Tecnologias de 
dispositivos de transmissão de informações digitais, e tecnologias de medição para fins de diagnóstico, tiveram destaque dentro do universo apurado. Já as tecnologias da informação e comunicação adaptadas para fins específicos foram as que mais cresceram nesse período. Além disso, foi possível observar a China como uma grande produtora de tecnologias de IoT para hospitais, já a Índia obteve destaque em termos de pesquisas científicas sobre essa temática. O trabalho permitiu ter uma visão das principais tendências da IoT para hospitais.

Palavras-chave: Hospitais inteligentes; IoT; Prospecção.

\begin{abstract}
Anchored in the internet of things (IoT) paradigm, Smart Hospitals have emerged as an innovative proposal for hospitals, where the functions of diagnosis, treatment, management and decision are integrated. In view of the great problems that hospitals around the world have faced, the need for an intelligent environment within the hospital context has been urgent, and the benefits brought by IoT for this sector are numerous. Understanding the importance of this theme, this work proposed to carry out a technological prospection study based on patents and scientific articles, in order to investigate technologies based on the IoT paradigm with application in hospital environments. Thus, searches were carried out on the Questel-Orbit databases, for patent documents, and Scopus, for scientific articles. Through patent and bibliometric analyzes it was possible to build a mapping of technologies and scientific research on IoT with application in hospitals, within the time frame from 2009 to 2020. The results pointed to an exponential growth of patent deposits and publications of articles on this topic. Technologies for digital information transmission devices, and measurement technologies for diagnostic purposes, were highlighted within the universe investigated. Information and communication technologies adapted for specific purposes were the ones that grew the most in this period. In addition, it was possible to observe China as a major producer of IoT technologies for hospitals, while India has gained prominence in terms of scientific research on this topic. The work provided an insight into the main trends of IoT for hospitals.
\end{abstract}

Keywords: Smart hospitals; IoT; Prospection.

\title{
Resumen
}

Anclados en el paradigma del internet de las cosas (IoT), los hospitales inteligentes han surgido como una propuesta innovadora para los hospitales, donde se integran las funciones de diagnóstico, tratamiento, gestión y decisión. Ante los grandes problemas a los que se han 
enfrentado los hospitales de todo el mundo, la necesidad de un entorno inteligente dentro del contexto hospitalario ha sido urgente, y los beneficios que aporta IoT para este sector son numerosos. Entendiendo la importancia de este tema, este trabajo se propuso realizar un estudio de prospección tecnológica basado en patentes y artículos científicos, con el fin de investigar tecnologías basadas en el paradigma IoT con aplicación en entornos hospitalarios. Así, se realizaron búsquedas en las bases de datos Questel-Orbit, para documentos de patente, y Scopus, para artículos científicos. A través de análisis bibliométricos y de patentes fue posible construir un mapeo de tecnologías e investigación científica en IoT con aplicación en hospitales, en el marco temporal de 2009 a 2020. Los resultados apuntan a un crecimiento exponencial de los depósitos de patentes y publicaciones de artículos sobre este tema. Dentro del universo investigado se destacaron las tecnologías para dispositivos de transmisión de información digital y las tecnologías de medición con fines de diagnóstico. Las tecnologías de la información y la comunicación adaptadas a fines específicos fueron las que más crecieron en este período. Además, fue posible observar a China como un importante productor de tecnologías de IoT para hospitales, mientras que India ha ganado prominencia en términos de investigación científica sobre este tema. El trabajo proporcionó una idea de las principales tendencias de IoT para hospitales.

Palabras clave: Hospitales inteligentes; IoT; Prospección.

\section{Introdução}

Considerada, na atualidade, como uma das esferas prioritárias do desenvolvimento em muitos países, a área da assistência em saúde sofreu grande influência nos últimos anos na busca por um modelo de assistência médica completamente diferente. O crescimento potencial e exponencial das novas tecnologias na medicina, bem como o aumento de suas capacidades, o rápido desenvolvimento da tecnologia da informação (TI), a evolução e a implementação das ideias da $4^{\mathrm{a}}$ Revolução Industrial, foram alguns marcos que impulsionaram o avanço nesse setor (Ilin, Iliyanschenko \& Konradi, 2018).

Atrelado aos grandes avanços da IoT no cenário global, um dos temas que têm sido emergentes na atualidade é o dos Hospital Inteligentes (Smart Hospitals). Para Yu, Lu e Zhu (2012), os hospitais inteligentes são baseados na tecnologia da Internet das Coisas (IoT) e concebidos no vetor de sistemas de serviços de aplicativos, tratando-se de um novo conceito de hospital, que integra as funções de diagnóstico, tratamento, gestão e decisão. Para os autores, a IoT reúne recursos necessários, como percepção abrangente, transmissão confiável, 
processamento inteligente, etc., para fornecer uma plataforma de serviços técnicos para construção e implementação de hospitais inteligentes. Segundo Cabra et al. (2017), o campo da saúde pode ser um dos campos mais favoráveis para integrar tecnologias de IoT.

Dentre as diversas aplicações da IoT em hospitais, um dos exemplos principais é o monitoramento remoto de pacientes, que podem estar em casa ou em outros lugares e, ao mesmo tempo, conectados a diversos tipos de sensores - vestíveis, de controle de sinais vitais, pressão arterial, glicemia, oximetria, entre outros. Isso possibilita que dados sejam gerados, coletados e transmitidos em tempo real pare que médicos possam prontamente fazer interações, quando for necessário (Gonçalves, 2017).

No gerenciamento hospitalar, estudos de tecnologias baseadas na IoT tem investigado novas soluções aos registros eletrônicos do paciente, fazendo integração em nuvem (Riad, Hanza \& Yan, 2019). Outras pesquisas têm procurado modernizar o cuidado com o paciente como dispositivos para prever e/ou alertar quedas (Liu et al., 2018), uma preocupação pertinente em hospitais; camas de hospitais acionadas sob o comando de voz (Kajol et al., 2019); tecnologias para monitoramento das funções cardíacas para prevenção de ataques cardíacos (Majumder et al., 2016); e tecnologias de previsão de crises epiléticas (Lin et al., 2018).

Diante desse contexto de possibilidades que a IoT pode promover e potencializar os serviços médico-hospitalares, este trabalho, a partir de uma abordagem científica e tecnológica, tem o objetivo de conduzir um estudo prospectivo de tecnologias de IoT aplicadas em ambientes hospitalares, identificando países protagonistas nessa área, empresas e instituições que tem se destacado, além de áreas tecnológicas que tem sido mais emergentes dentro dessa temática.

A importância deste estudo perpassa pela própria importância da realização de um estudo prospectivo de um tema emergente. Utilizada nos últimos anos por governos e empresas privadas, a prospecção tecnológica tem se consolidado como uma ferramenta de inteligência competitiva. $\mathrm{O}$ monitoramento de tecnologias em base de patentes é um dos métodos de realização de um estudo prospectivo, em que seus resultados, em geral quantitativos, podem ser utilizados em processos decisórios, levando em consideração as avaliações qualitativas de especialistas (Mendes, 2012). Além disto, os documentos de patentes possuem um enorme valor em termos de informação, uma vez que disponibilizam ao público informação qualificada, referente a todos os campos tecnológicos indexados pela Classificação Internacional de Patentes (OMPI, 2014). O levantamento bibliométrico em bases de periódicos científicos também permite investigar o estado da arte de tecnologias 
específicas, levando em consideração que os periódicos são o principal meio de comunicação da ciência.

\section{IoT e Hospitais Inteligentes}

A IoT é um novo paradigma que oferece um conjunto de possibilidades em serviços para a próxima geração de inovações tecnológicas. A integração perfeita entre o mundo cibernético com o mundo físico faz as oportunidades de aplicações em IoT serem praticamente ilimitadas (Colakovic \& Hadzialic, 2018). Avanços tecnológicos recentes, tais como, redes de sensores sem fio, comunicação móvel e computação ubíqua, possibilitaram o surgimento da IoT. Ainda assim, para que esse paradigma seja amplamente disseminado, uma série de obstáculos precisam ser superados, principalmente com relação ao desenvolvimento de aplicações e a alta heterogeneidade de hardware e software desse ambiente, inerente a sua própria diversidade de tecnologias (Pires et al. 2015).

O termo Internet das coisas, criado pelo britânito Kevin Ashton em 1999, apareceu pela primeira vez no centro do Auto-ID ${ }^{1}$, no Instituto de Tecnologia de Massachusetts. O grupo de pesquisa RFID (Radio Frenquency Identifcation) define IoT como a rede mundial de objetos interconectados, endereçáveis de maneira única, com base em protocolos de comunicação padrão. Hoje o conceito que incorpora campos tradicionais como sistemas embarcados, sistemas de controle e automação, redes de sensores sem fio para facilitar a comunicação dispositivo a dispositivo, através da Internet, possui aplicações para usuários particulares e empresariais (Kulkarne \& Sathe, 2014).

Em um dos últimos levantamentos (Figura 1) realizados pela Gartner em 2019, focado especificamente em tecnologias relacionadas à Internet das Coisas, pode ser observado o nível de maturidade que temas ligados a IoT já alcançaram. De acordo com a instituição, alguns conceitos que já saíram do pico, como veículos autônomos, plataformas de IoT, arquitetura de IoT e processamento de fluxo de eventos começam a entrar em declínio de expectativas para a fase de adesão pela comunidade. Já outros conceitos, como ativos de localização interna, integração de IoT, gerenciamento de serviços de IoT, já se encontram em fase de adesão da comunidade. Vale destacar que outros termos começam a se associar à IoT, em fase de descoberta da inovação, como Blockchain e Master Data Management.

\footnotetext{
${ }^{1}$ Laboratório de pesquisa em RFID e tecnologias emergentes em detecção do MIT.
} 
Figura 1 - Cycle Hyper sobre a Internet das Coisas em 2019.

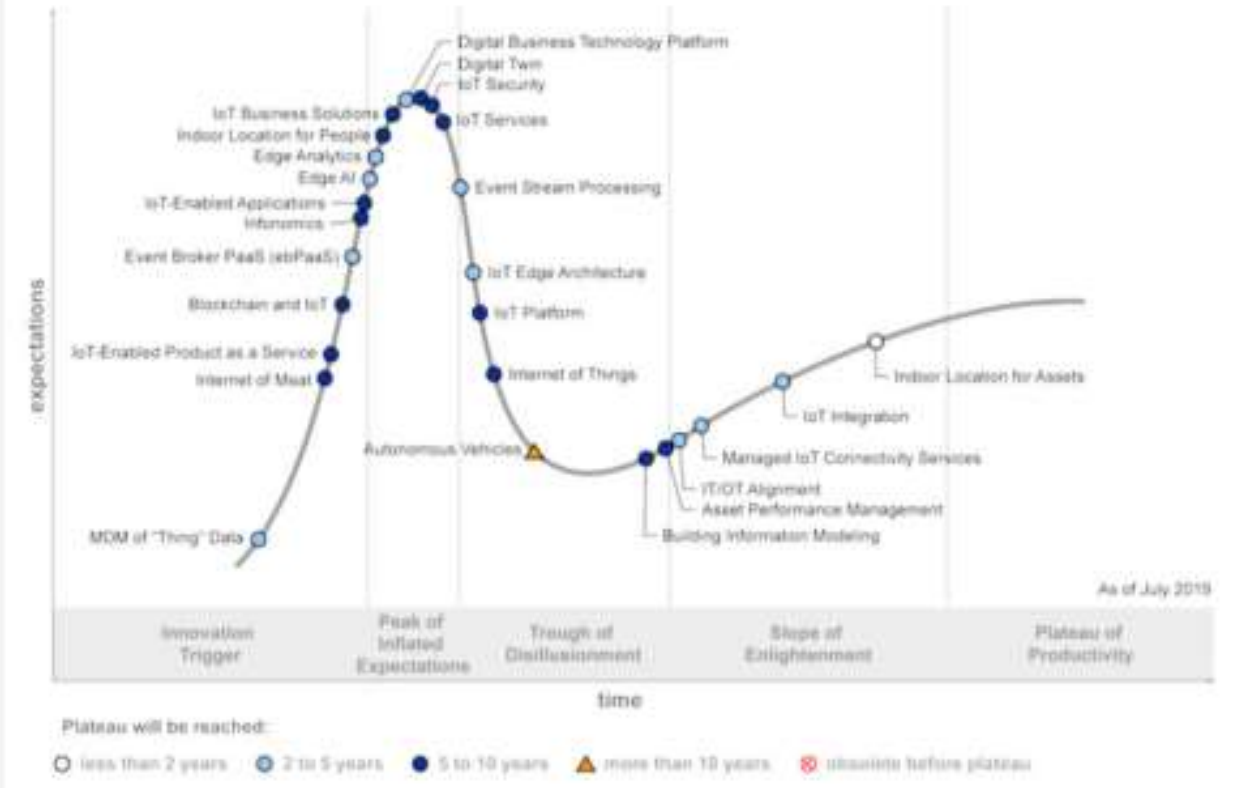

Fonte: Gartner, (2019).

Cidades inteligentes, casas inteligentes, controle de poluição, economia de energia, transporte inteligente, indústrias inteligentes são algumas das transformações decorrentes da IoT (Kumar, Tware \& Zybler, 2019). As vantagens de dispositivos inteligentes interconectados, numa eficiente interação homem-máquina, são inúmeras. Por exemplo, no âmbito imobiliário e habitacional, dispositivos conectados a aplicativos de smartphones podem abrir e fechar janelas, ligar chuveiros, destrancar portas e autorregular a temperatura, promovendo segurança e autonomia. $\mathrm{Na}$ área da saúde, o monitoramento constante e eficiente e uma interação mais eficaz entre paciente e médico será viável, possibilitando também o controle e a prevenção de epidemias (Magrani, 2018).

De acordo com Raj et al. (2017) os hospitais inteligentes são um processo médico que faz o hospital funcionar de maneira inteligente e não apenas digital. Esse processo, muito otimizado e desenvolvido para gerenciar a infraestrutura do hospital, é habilitado por uma infraestrutura interconectada e digitalizada, subjacente dos ativos de rede, com objetivo de manter serviços e insights valiosos, obter maior atendimento ao paciente, experiência e potência operacional. Na visão da ENISA (2016), esse modelo de hospital depende de processos otimizados e automatizado para melhorar os procedimentos de atendimento ao paciente existentes e introduzir novos recursos, sendo construído em um ambiente de ativos interconectados da tecnologia da informação e comunicação, principalmente com base na IoT. 
A proposta dos hospitais inteligentes é fornecer um conjunto mais restrito de serviços de alto valor em um ecossistema mais amplo de entidades, já que, em geral, algumas dessas entidades podem não estar associadas à prestação de serviços de saúde. Podem fazer parte desse grande ecossistema, como apresentado na Figura 2, clínicas, academias, farmácias, centros laboratoriais e até mesmo a residência do paciente. Com esse ecossistema de compartilhamento de dados e informações, que está fundamentalmente centrado no paciente, é possível ter como resultados: prestação de serviços de saúde mais convenientes e eficazes, menor custo médico, melhor qualidade de serviço — devido a funções —, e responsabilidades claras (CHEN et al., 2019).

Figura 2 - Ecossistema inteligente de entidades centrada no paciente.

\section{COMPARTILHAMENTO DE DADOS/INFORMAÇĀO}

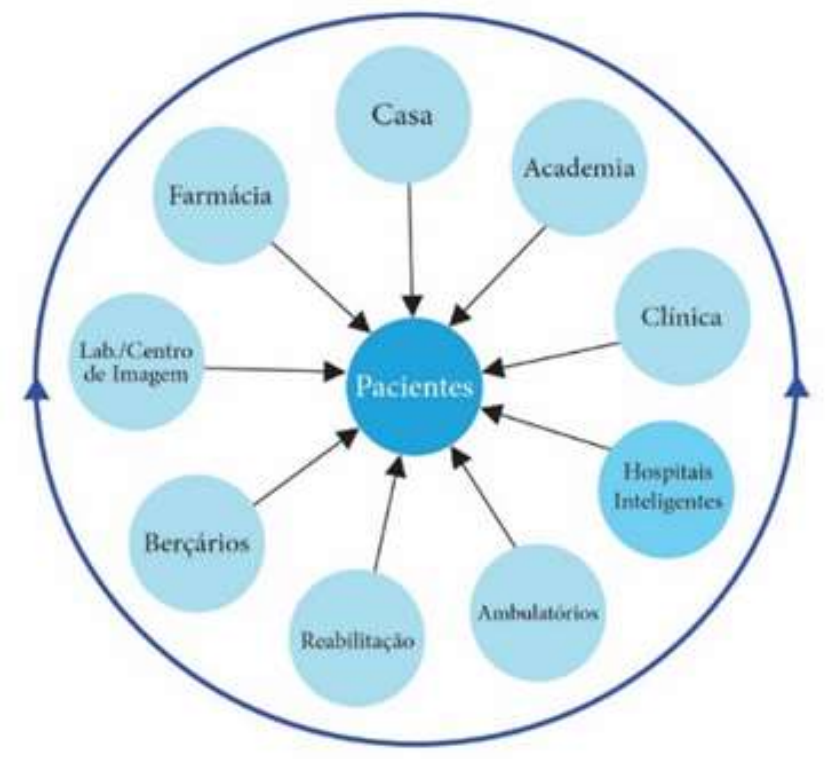

Fonte: Adapartado de Chen et al., (2019).

Para Takhare e Khire (2014) as organizações médicas enfrentam diversas dificuldades no gerenciamento de informações clínicas, financeiras e operacionais. Mudar o gerenciamento hospitalar para um sistema inteligente de gerenciamento demanda dispositivos inteligentes, que ajudarão a organização com o fluxo contínuo de dados entre sistemas e unidades de negócios diferentes. Nos níveis macroscópico e microscópico, essas tecnologias poderão oferecer melhor atendimento e maior satisfação aos pacientes, prestadores de cuidados e atendentes.

Não é um padrão definido de hospitais inteligentes que determinará se uma instalação é inteligente ou não, e sim uma abordagem, que através das ferramentas de tecnologias 
inteligentes, encontra soluções reais para desafios reais. Com a execução adequada e atenção especializada à interoperabilidade de arquitetura aberta, os operadores de uma instalação hospitalar estarão preparados para aplicar novas tecnologias aos desafios atuais e vindouros (ANIXTER, 2018). Ademais, o que determina se um hospital é inteligente é a disponibilidade e o uso de sistemas e dispositivos substancialmente interconectados que suscitam à inteligência geral (ENISA, 2016).

Embora a área da saúde tenha recebido a IoT de maneira lenta nos últimos tempos, no centro da pandemia da COVID-19, que o mundo atravessa em 2020, esse cenário tem mudado rapidamente. Com o setor da saúde em evidência, a demanda por aplicações de IoT, como consultas de telessaúde, diagnóstico digital, monitoramento remoto e assistência de robôs está aumentando. Na visão de diretores de tecnologia da saúde, há um número crescente de pacientes sendo tratatados remotamente em todo o mundo, com um aumento significativo de consultas online, impulsionando a digitalização dos processos tradicionais em setores médicos (IoT ANALYTICS, 2020). É provável que muitas dessas soluções possam ultrapassar a crise atual, permanecendo e criando disrupções no setor médico-hospitalar.

\section{Metodologia}

Quanto a sua natureza ou finalidade, esta pesquisa pode ser classificada como uma pesquisa aplicada. Esse tipo de pesquisa é caracterizado por gerar conhecimentos para aplicação prática, voltados a solução de problemas específicos, envolvendo verdades e interesses locais (Silva \& Menezes 2001). Do ponto de vista dos objetivos específicos, esta pesquisa se posiciona como uma pesquisa exploratória. Em geral, este tipo de pesquisa é realizado quando se há pouco conhecimento acumulado e sistematizado sobre um tema (Gil, 2002). Quanto a abordagem este trabalho combina aspectos quantitativos e qualitativos, utilizando métodos estatísticos utilizados pelos estudos métricos da informação, especificamente da patentometria e bibliometria.

A primeira etapa deste estudo consistiu na fase de pré-prospecção, na qual foi realizada um estudo exploratório acerca dos temas da IoT, hospitais inteligentes e metodologias de prospecção. Para construção da prospecção, este estudo considerou documentos de patentes e artigos científicos. Para o levantamento de patentes foi escolhido o sistema de inteligência Questel-Orbit, e para o estudo bibliométrico de artigos foi utilizada a plataforma Scopus. Para a realização das buscas nas referidas bases, foram definidas as 
strings (palavras-chaves) que remetessem características fundamentais e essenciais da tecnologia que está sendo investigada, delimitando o corte temporal de 2009 a 2020.

No sistema Orbit a string foi aplicada nos campos título, resumo e reivindicações. $\mathrm{Na}$ plataforma Scopus a string foi aplicada nos campos título, resumo e palavra-chave. Foram utilizados operadores booleanos (OR e AND), e caracteres curinga (“*” e “?”) para que possíveis variações de palavras também fossem incluidas. As buscas foram realizadas entre os meses de julho de 2020 até setembro de 2020. A combinação das strings utilizadas são apresentadas no Quadro 1.

Quadro 1 - Estratégia de busca com as strings aplicada nas bases de dados

\begin{tabular}{|c|c|}
\hline SCOPUS & ORBIT \\
\hline $\begin{array}{c}\text { (“smart hospital*”) OR ("intel?igent hospital*”) } \\
\text { OR ((hospital* OR medic* OR clinic*) AND (iot } \\
\text { OR “internet of thing*”) }\end{array}$ & $\begin{array}{c}\text { (smart hospital } *) \text { OR (intel? igent hospital } *) \text { OR } \\
((\text { hospital } * \text { OR medic* OR clinic } *) \text { AND (iot OR } \\
(\text { internet of thing*)) }\end{array}$ \\
\hline $\begin{array}{l}\text { O Scopus é o maior banco de dados de resumos e } \\
\text { citações da literatura com revisão por pares, } \\
\text { abrangendo revistas científicas, livros, processos } \\
\text { de congressos e publicações do setor, incluindo } \\
\text { ferramentas de análise e verificação da pesquisa. }\end{array}$ & $\begin{array}{l}\text { O sistema Orbit reúne documentos de patentes de } 87 \\
\text { escritórios nacionais e } 6 \text { escritórios regionais (EPO, } \\
\text { WIPO, OAPI, ARIPO, EAPO e CGC), além de } \\
\text { possuir ferramentas robustas que possibilitam } \\
\text { diversas análises de informações contidas nos } \\
\text { documentos de patente. }\end{array}$ \\
\hline
\end{tabular}

Fonte: Autores, (2020).

O tratamento e refino dos dados foi utilizado para extrair documentos e registros que não se aplicam a este estudo. Assim, tanto para os documentos de patentes como para os artigos científicos, foi feita a leitura do título e do resumo, e dentro das próprias bases foram utilizadas ferramentas de exclusão e criação de listas para separar os resultados apurados.

Numa análise prospectiva os primeiros resultados esperados, é responder três principais questões: quando (refere-se a análise da tendência histórica), onde (refere-se análise geográfica), e o quem (procura identificar os detentores de tecnologia) (Alencar, 2008). Assim, utilizando os recursos de análises das bases utilizadas e também dos programas Microsoft Excel e VosViewer, diversas análises quantitativas e qualitativas foram realizadas identificando países, empresas/instituições, áreas tecnológicas e analisando a própria evolução da tecnologia ao longo do tempo. 


\section{Resultados e Discussão}

\subsection{Análise Patentária}

Este estudo recuperou um total de 3007 famílias de patentes na base Orbit, considerando o corte temporal de 2009 a 2020 e utilizando a estratégia de busca que é apresentada no Quadro 2. Com o tratamento dos dados, foram apuradas 2015 famílias de patentes para realização das análises.

Quadro 2 - Resultado da estratégia de busca na base Questel-Orbit.

\begin{tabular}{|c|c|}
\hline \multicolumn{2}{|r|}{ Questel Orbit } \\
\hline String & $\begin{array}{l}\text { (smart hospital } * \text { ) OR (intel?igent hospital } *) \text { OR ((hospital } * \text { OR medic* } \\
\text { OR clinic*) AND (iot OR (internet of thing } *))\end{array}$ \\
\hline Período & $2009-2020$ \\
\hline Resultado apurado & 2015 famílias de patentes \\
\hline
\end{tabular}

Fonte: Autores, (2020).

Considerando o ano de primeiro depósito das famílias de patentes levantadas, o gráfico da Figura 3 permite uma visão da evolução desses depósitos ao longo dos últimos dez anos, tendo expressividade, principalmente, entre os anos de 2017 e 2019, com uma média de 385 depósitos de patentes por ano. Apesar do decrescimento no número de depósitos no ano de 2019 e 2020, é considerado que os dados estavam incompletos, tendo em vista os prazos protocolares nos diversos escritórios de patentes, que em geral demandam tempo para publicitar as informações devido, principalmente, ao período de sigilo que é imposto à patente, após o seu depósito.

Figura 3 - Número de famílias de patentes depositadas por ano.

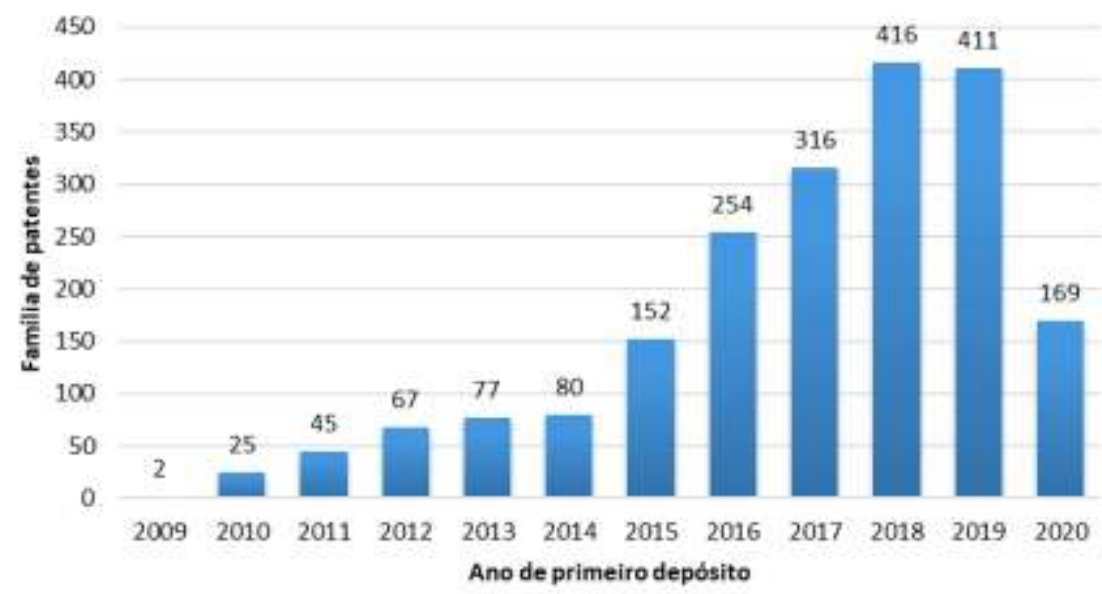

Fonte: Autores, a partir da base Questel-Orbit (2020). 
Esse crescimento indica a forte tendência dessas tecnologias no setor da saúde. $\mathrm{Na}$ visão da i-Scoop (2020), empresa de consultoria em transformação digital, a vastidão do ecossistema da saúde faz com que a lista de aplicações da IoT nessa área seja quase que infinita. Com tecnologias que vão desde a saúde pessoal, biossensores e camas inteligentes, até aos monitoramentos remotos e as várias especializações e atividades de saúde e até mesmo tratamentos de doenças.

Em pesquisa realizada pela IoT Analytics (2020), em que foram analisadas 1414 publicações sobre projetos de IoT, a área da saúde já ocupa a sexta posição, duas posições a mais que o último levantamento realizado em 2018, ultrapassando outras áreas de grande importância para a sociedade, como agricultura, cadeia de mantimentos e a construção civil, apresentando assim uma tendência de crescimento. Para a empresa, o atual cenário de pandemia em 2020 aumentou a demanda por soluções de IoT para saúde, intensificando os projetos de aplicações de telessaúde, diagnóstico digital, monitoramento remoto e assistência de robôs.

Os dados investigados neste estudo apresentaram 15 países com depósitos de patentes de tecnologias de IoT hospitalar, além dos escritórios da World Intellectual Property Organization (WIPO) e do European Patent Office (EP) que também estão incluídos nesta análise. A Figura 4 apresenta esses países e representações, e o número de pedidos prioritários depositados nos vários escritórios nacionais. Esse gráfico demonstra as estratégias de patentes dos requerentes, além de identificar onde as principais equipes de pesquisas estão localizadas, sendo um excelente indicador de $\mathrm{P} \& \mathrm{D}$, pois, em geral, a maioria dos participantes registra pedidos de patentes prioritárias localmente.

Figura 4 - Quantitativo de famílias de patentes por país de prioridade.

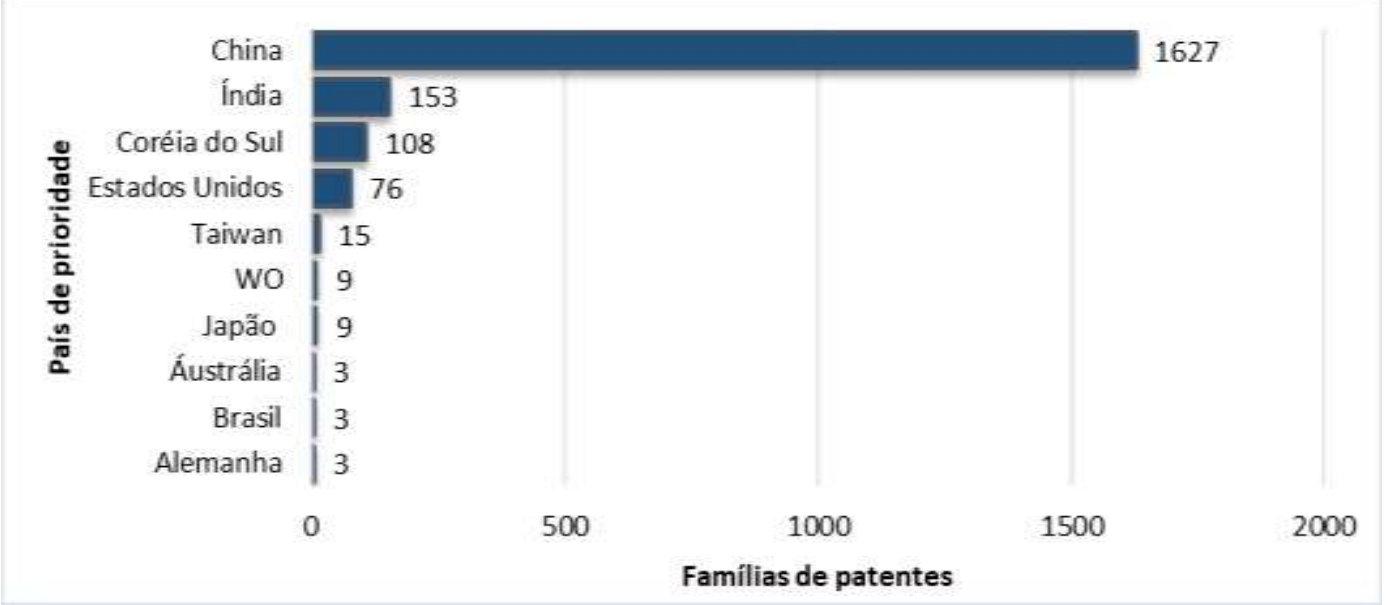

Fonte: Autores, a partir da base Questel-Orbit (2020). 
Research, Society and Development, v. 9, n. 11, e50791110081, 2020

(CC BY 4.0) | ISSN 2525-3409 | DOI: http://dx.doi.org/10.33448/rsd-v9i11.10081

É notória a disparidade chinesa em termos de depósito de patentes no campo da IoT hospitalar, sendo seu quantitativo expressivamente maior que a soma de todas as patentes dos demais países e representações de patentes, contabilizando um total 1627 famílias de patentes. Em segundo lugar, a Índia apresenta 153 depósitos de famílias de patentes, seguido da Coréia do Sul, Estados Unidos e Taiwan com 108, 76 e 15 famílias de patentes respectivamente. Os demais países e representações listados apresentaram menos de 10 depósitos de patentes cada.

A China tem investido grandes quantias de recursos para impulsionar o desenvolvimento da chamada intelligent healthcare (saúde inteligente), sendo um dos pilares centrais da nova reforma da saúde no país, na qual a expectativa é solucionar o conflito do país entre recursos limitados de assistência médica e grande população de pacientes. Com muitos desafios, a indústria de saúde inteligente na China tem um grande potencial e está crescendo muito rápido. Centenas de empresas locais têm se desenvolvido muito e algumas delas podem fornecer tecnologias avançadas, de hospitais inteligentes a um sistema regional de saúde. A explosão de dispositivos portáteis, voltados à área da saúde, é outro indicador do potencial chinês, sendo possível que a China se torne o maior mercado do mundo na indústria da saúde inteligente (Zheng \& Rodriguez-Monroy, 2015).

Além disso, de acordo com Chen et al. (2018), uma das características fundamentais para o desenvolvimento da IoT, de modo geral, na China, são as políticas governamentais. Apesar de empresas privadas e instituições acadêmicas também possuírem papeis importantes no motor da inovação e captutra de mercado, a área da IoT é extremamente beneficiada pelas políticas governamentais e pelo apoio financeiro que tem recebido na China. Enquanto os mandatos emitidos pelo Estado orientam os ministérios do governo a coordenar políticas em busca de uma adoção mais rápida e ampla da IoT, os recursos do governo são especialmente organizados para P\&D.

$\mathrm{O}$ ranking de empresas e instituições requerentes de patentes relacionadas a IoT com aplicação em hospitais é apresentado na Figura 4. Dessas dez empresas e instituições, sete são chinesas (Shenzhen Allwins, Fudan University, Shandong Walihon, Shangai Chengyi, Shandong Quinchengm Hangzhou Xiaochuang e Ewell Tech), duas sul-coreanas (Hanyang University e Jeong Eui) e uma estadunidense (IBM). Pode-se observar também a predominância de empresas de tecnologia, constando duas universidades sendo uma delas um hospital universitário como requerente de depósito de patentes. Esse gráfico é um bom indicador de inventividade dos atuais competidores ativos no mercado. 
Research, Society and Development, v. 9, n. 11, e50791110081, 2020

(CC BY 4.0) | ISSN 2525-3409 | DOI: http://dx.doi.org/10.33448/rsd-v9i11.10081

Figura 5 - Quantitativo de famílias de patentes por requerentes (empresas/instituições).

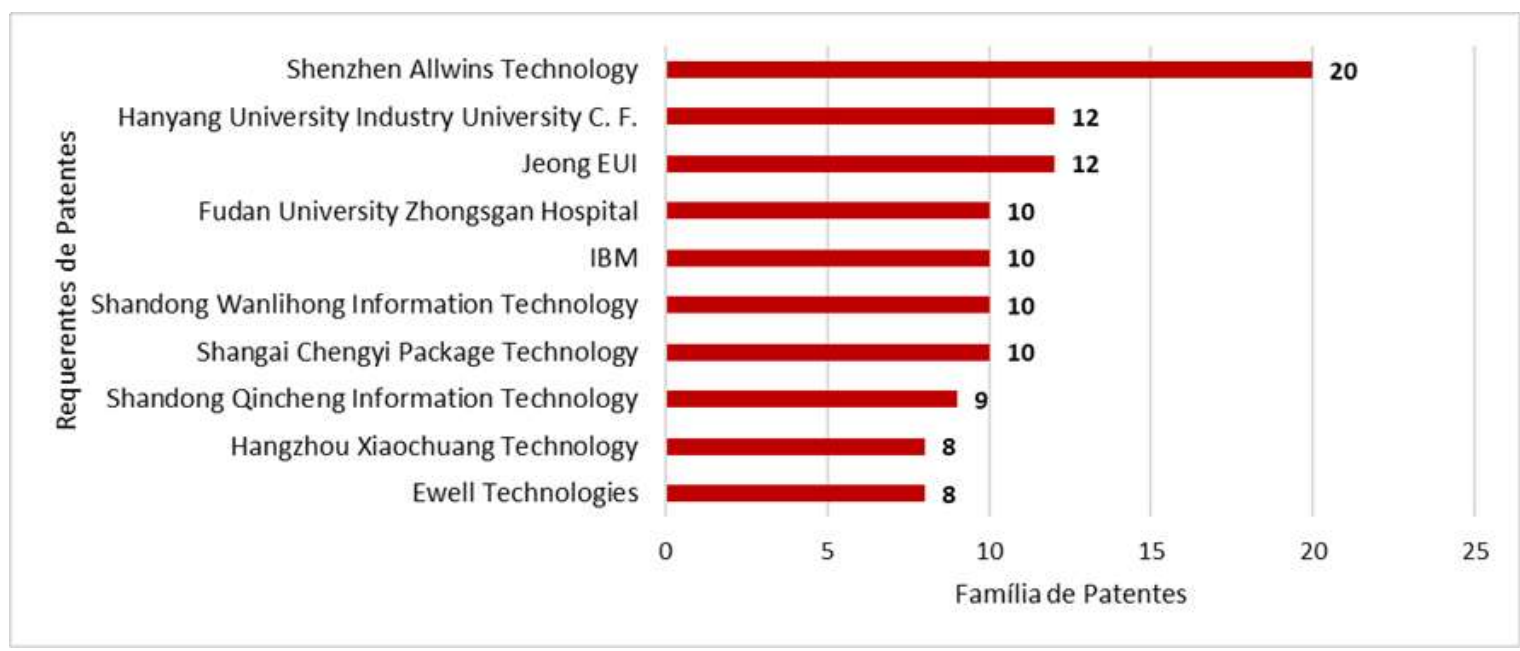

Fonte: Autores, a partir da bases Questel-Orbit (2020).

A primeira posição é ocupada pela empresa chinesa Shenzhen Allwins Technology, líder de mercado de produtos de rede, a empresa produz roteadores, conversores de fibra, placas de rede sem fio e outros produtos. A Coréia do Sul é representada nesse ranking por duas instituições/empresas: a Hanyag University e Jeong Eui, ambas com 12 famílias de patentes, as quais são parceiras na titularidade. A gigante norte-americana IBM, aparece na quinta posição com 15 depósitos de famílias de patentes. Consagrada como a maior empresa de tecnologia da informação do mundo, essas patentes representam apenas cerca de um centésimo do enorme portfólio de patentes da IBM.

A Classificação Internacional de Patentes (IPC) é uma excelente ferramenta para classificar o conteúdo técnico dos documentos de patentes em áreas tecnológicas. Observando a Figura 6, pode-se analisar a evolução dessas áreas tecnológicas ao longo do tempo, podendo destacar crescimentos e decrescimentos. 
Figura 6 - Evolução dos principais códigos IPCs entre os anos de 2009-2020.

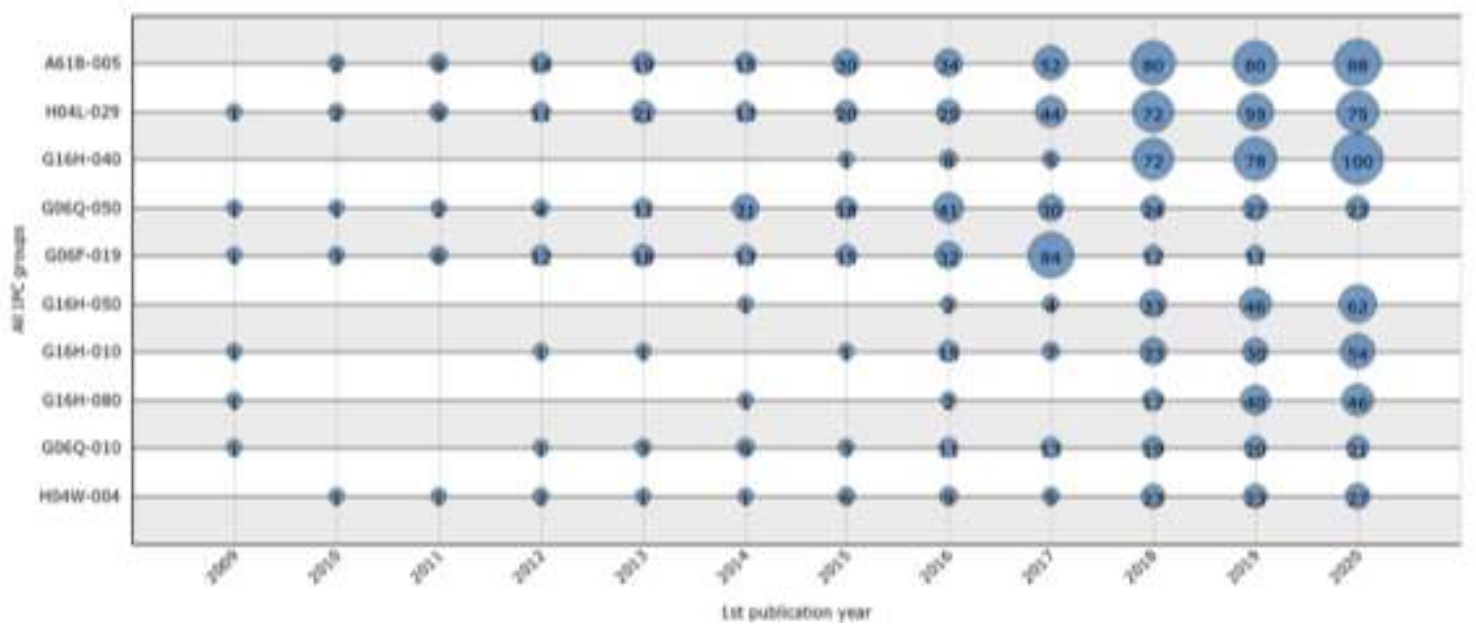

Fonte: Questel-Orbit (2020).

O grupo A61B-005 apresenta o maior número de patentes dentro do universo analisado, com 423 famílias de patentes, que classifica tecnologias de diagnóstico, sendo especificamente sobre medições para fins de diagnóstico. Em seguida se destaca o grupo H04L-029, com 352 famílias de patentes, esse código se refere a tecnologias de transmissão de informações digitais (disposições, aparelhos, circuitos ou sistemas). As descrições dos principais códigos IPC analisados neste estudo são apresentadas no Quadro 3.

Quadro 3 - Descrições dos principais códigos IPCs das patentes de IoT com aplicação em hospitais.

\begin{tabular}{|c|l|}
\hline $\begin{array}{c}\text { GRUPO DE } \\
\text { IPC }\end{array}$ & \multicolumn{1}{c|}{ DESCRIÇÃO } \\
\hline A61B-005 & DIAGNÓSTICO; CIRURGIA; IDENTIFICAÇÃO - Medição para fins de diagnóstico. \\
\hline H04L-029 & TRANSMISSÃO DE INFORMAÇÕES DIGITAIS - Disposições, aparelhos, circuitos ou sistemas. \\
\hline G16H-040 & $\begin{array}{l}\text { INFORMÁTICA DA SAÚDE - TIC especialmente adaptadas para a gestão ou administração de } \\
\text { recursos ou instalações de saúde. }\end{array}$ \\
\hline G06Q-050 & $\begin{array}{l}\text { SISTEMAS OU MÉTODOS DE TRATAMENTO DE DADOS - Sistema ou método de tratamento } \\
\text { de dados, especialmente adaptado para setores específicos (por exemplo, administração). }\end{array}$ \\
\hline G06F-019 & $\begin{array}{l}\text { PROCESSAMENTO DE DADOS DIGITAIS ELÉTRICOS - Equipamento ou métodos de } \\
\text { computação digital ou de processamento de dados, especialmente adaptados para aplicações } \\
\text { específicas. }\end{array}$ \\
\hline G16H-050 & $\begin{array}{l}\text { INFORMÁTICA DA SAÚDE - TIC especialmente adaptadas para diagnóstico médico, simulação } \\
\text { médica ou mineração de dados médicos; TIC especialmente adaptadas para detectar, monitorar ou } \\
\text { modelar epidemias ou pandemias. }\end{array}$ \\
\hline G16H-010 & $\begin{array}{l}\text { INFORMÁTICA DA SAÚDE } \\
\text { TIC especialmente adaptadas para o manuseio ou processamento de dados médicos ou de saúde } \\
\text { relacionados ao paciente. }\end{array}$ \\
\hline G06Q-010 & $\begin{array}{l}\text { INFORMÁTICA DA SAÚDE - TIC especialmente adaptado para facilitar a comunicação entre } \\
\text { médicos ou pacientes, por exemplo, para diagnóstico colaborativo, terapia ou monitoramento de } \\
\text { saúde. }\end{array}$ \\
\hline SISTEMAS OU MÉTODOS DE TRATAMENTO DE DADOS - Administração; Gestão. \\
\hline
\end{tabular}

Fonte: Autores (2020). 
Além dos dois principais códigos de IPCs já destacados anteriormente, vale destacar também os códigos G16H-050, G16H-010, e G16H-080, todos da mesma família G16H (informática da saúde), que se referem a tecnologias da informação e comunicação (TIC) especialmente adaptadas para fins específicos. Esses códigos representam tecnologias que apresentaram crescimento em seus depósitos, em especial nos últimos quatro anos. Isso pode revelar a informatização hospitalar que tem se intensificado nos últimos anos, com o intuito de digitalizar o máximo de informações e recursos nas instalações de saúde. O maior decrescimento foi notado no grupo G06F-019, relacionados a processamento de dados digitais elétricos, e especificamente a equipamentos ou métodos de computação digital ou de processamento de dados, especialmente adaptados para aplicações específicas.

A Figura 7 apresenta o gráfico landscape com os principais clusters tecnológicos das patentes de IoT com aplicação em ambientes hospitalares. Pode ser observado as áreas com a coloração mais forte, onde há uma grande concentração de tecnologias relacionadas a conceitos em comum.

Figura 7 - Panorama dos principais clusters de tecnologias de IoT com aplicação hospitalar.

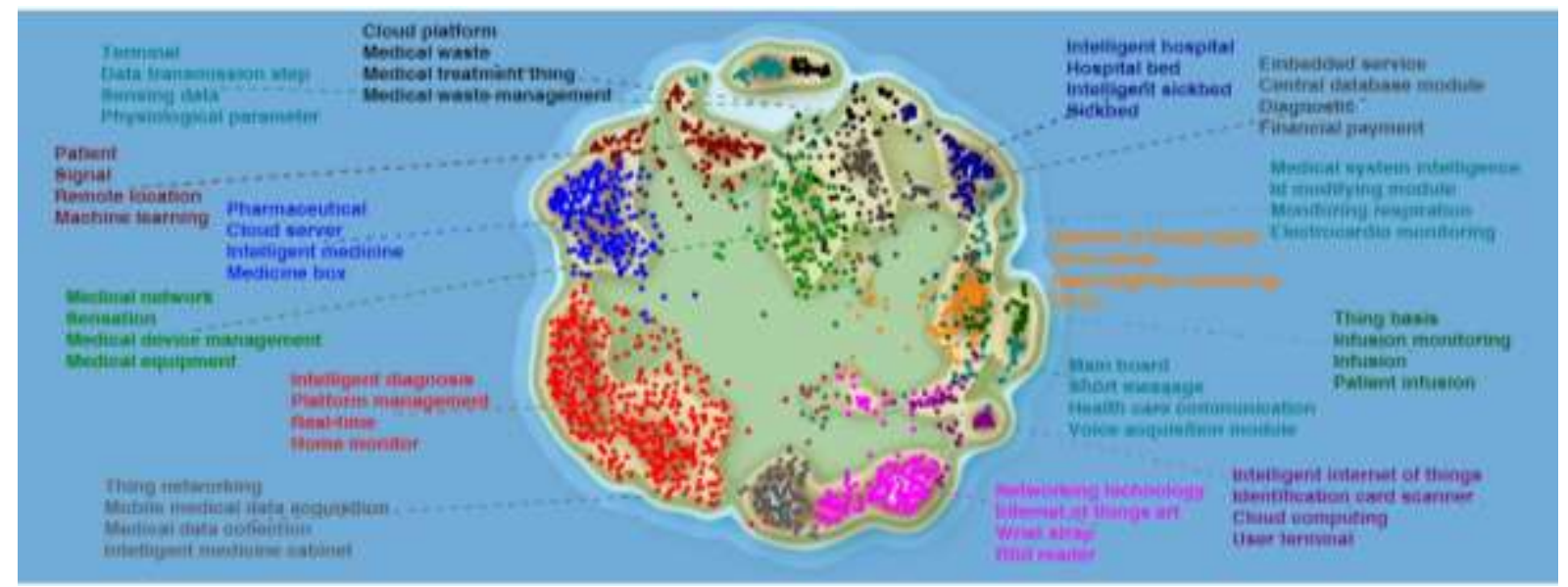

Fonte: Autores, a partir da base Questel-Orbit (2020).

Vale destacar as áreas: inteligente diagnosis, platform management, real-time, home monitor, que podem envolver tecnologias inteligentes para diagnóstico para monitoramento em tempo real; pharmaceutial, cloud server, inteligente medicine, medicine box, que podem se tratar de tecnologias para armazenamento e/ou gerenciamento de medicamentos, utilizando recursos compartilhados na núvem; networking technology, internet of things art, wrist strap e Rfid reader, que apontam para tecnologias de rede em forma de braceletes, munhequeiras ou pulseiras, utilizando recursos tecnológicos da RFID. 
Também é possível notar áreas com baixa sobreposição, o que pode indicar oportunidades de exploração de mercado dentro de determinados conglomerados tecnológicos. Pode-se destacar as áreas: medical waste, medical waste management, medical treatment thing, que indicam tecnologias de gerenciamento do descarte do lixo hospitalar; hospital bed, inteligente sickbed, sickbed, que se tratam de camas hospitalares inteligentes.

São diversos os dispositivos e aplicativos móveis que têm desempenhado um importante papel no rastreamento e prevenção de doenças crônicas para muitos pacientes e seus médicos. A aliança do desenvolvimento da IoT com tecnologias de telemedicina e telessaúde tem feito surgir uma nova abordagem: a Internet of Medical Things (IoMT), em que diversos tipos de monitoramento são possíveis, incluindo medições médicas comuns, como temperatura corporal, nível de glicose e leituras de pressão arterial (MOBIDEV, 2020).

Uma realidade recente que exemplifica a aplicação do telemonitoramento remoto de pacientes é a situação de pacientes com condições crônicas que precisam ser poupados em estar no ambiente hospitalar por conta da COVID-19, embora precisem continuar seus tratamentos. Assim, por meio dos cuidados de saúde habilitados para a IoT, poderão ser monitorados remotamente e com precisão na segurança de sua própria casa (HEALTHCARE GLOBAL, 2020b). Previsões nessa área já apontam que o monitoramento remoto do paciente eliminará a necessidade de muitas visitas ao médico, e além disso, os dados disponibilizados aos profissionais de saúde lhes permitirão ajustar os regimes de tratamento diariamente (Digital Information World, 2020).

\subsection{Análise bibliométrica}

O levantamento bibliométrico realizado na plataforma Scopus, recuperou um total de 2002 artigos científicos, publicados entre os anos de 2009 e 2020. Na busca foram utilizadas as mesmas palavras-chave do levantamento patentário. Após o tratamento do total de artigos recuperados, foram apurados 1557 artigos que foram separados para análise. Essa estratégia de busca está apresentada no Quadro 4. 
(CC BY 4.0) | ISSN 2525-3409 | DOI: http://dx.doi.org/10.33448/rsd-v9i11.10081

Quadro 4 - Resultado da estratégia de busca na plataforma Scopus

\begin{tabular}{|l|l|}
\hline \multicolumn{2}{|c|}{ SCOPUS } \\
\hline String & $\begin{array}{l}\text { ("smart hospital*”) OR ("intel?igent hospital*”) OR ((hospital* OR } \\
\text { medic* OR clinic*) AND (iot OR "internet of thing*”) }\end{array}$ \\
\hline Tipo de documento & Artigos \\
\hline Período & 2009-2020 \\
\hline Resultados apurados & 1557 artigos \\
\hline
\end{tabular}

Fonte: Autores, (2020).

De acordo com o gráfico da Figura 8, estudos científicos sobre o tema da IoT com aplicações hospitalares tiveram suas primeiras publicações em meados anos de 2009 e 2010, sendo a primeira publicação nesse período em 2009, na Índia. Apenas a partir de 2014 começa a ser notada uma intensificação nas pesquisas nessa área, que pode ser evidenciada com o crescimento exponencial que se deu a partir desse ano nas publicações científicas sobre IoT relacionada à área médico-hospitalar.

Figura 8 - Número de artigos por ano de publicação.

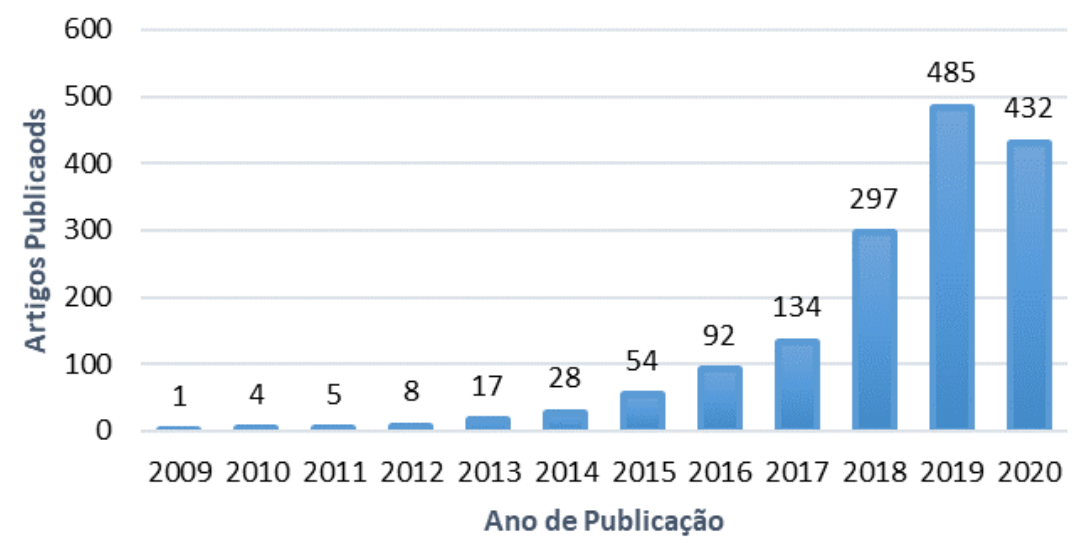

Fonte: Autores, a partir da base Scopus (2020).

A Figura 9 traz os dez principais países em termos de publicação científica sobre IoT aplicada em hospitais. Com 441 publicações científica, a Índia aparece na primeira posição nesse hanking. Em seguida aparecem China com 299 publicações, Estados Unidos com 205, Coréia do Sul com 136, Reino unido com 116, Arábia Saudita com 76, Taiwan com 63, Paquistão com 54, Itália com 53, e Espanha com 51 publicações. 
Figura 9 - Número de artigos por país de publicação.

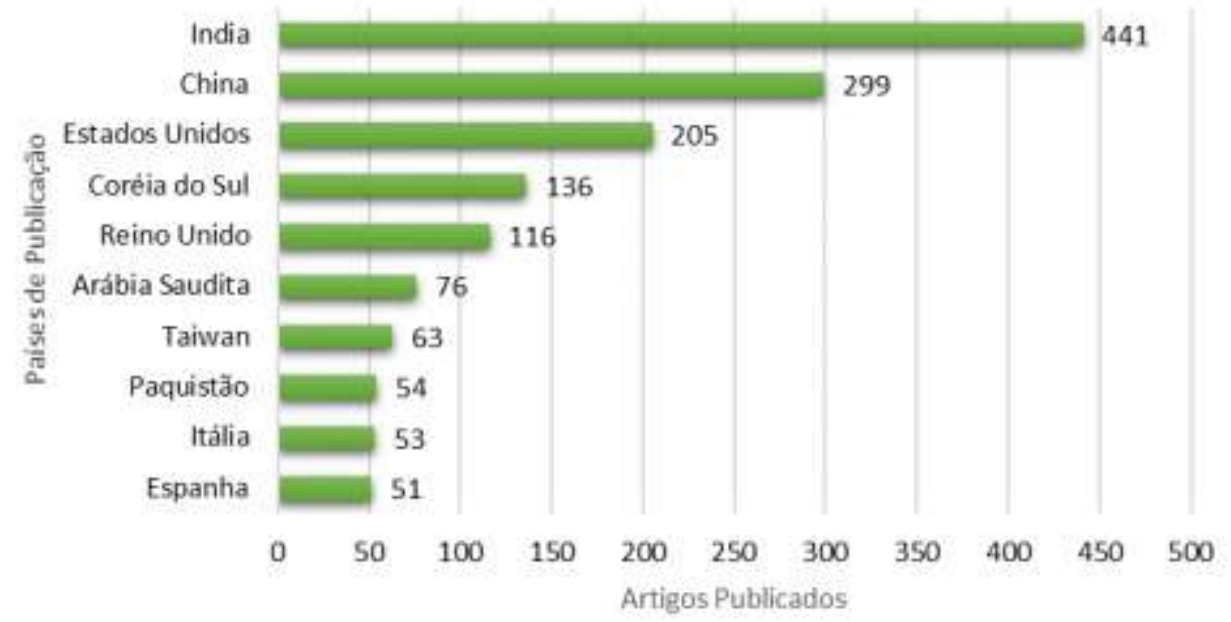

Fonte: Autores, a partir da base Scopus (2020).

Vale destacar que o governo da Índia implantou, em 2015, uma política específica de IoT, que dentre os seus objetivos estabelece a mobilização de pesquisas e desenvolvimentos relacionados à IoT de maneira eficaz, no intuito de impulsionar todos os assistentes tecnológicos do país. Um dos projetos prioritários do governo indiano é o Digital India Program, com o intuito de promover a digitalização e tornar a Índia um país com poder digital e economia do conhecimento, fornecendo a motivação necessária para a expansão do ecossistema de produtividade da IoT no país (Chatterjee \& Kar, 2018).

A Figura 10 apresenta a rede de conexões de palavras-chave com as principais dos artigos científicos levantados neste estudo.

Figura 10 - Rede de palavras referente as principais palavras-chaves dos artigos publicados.

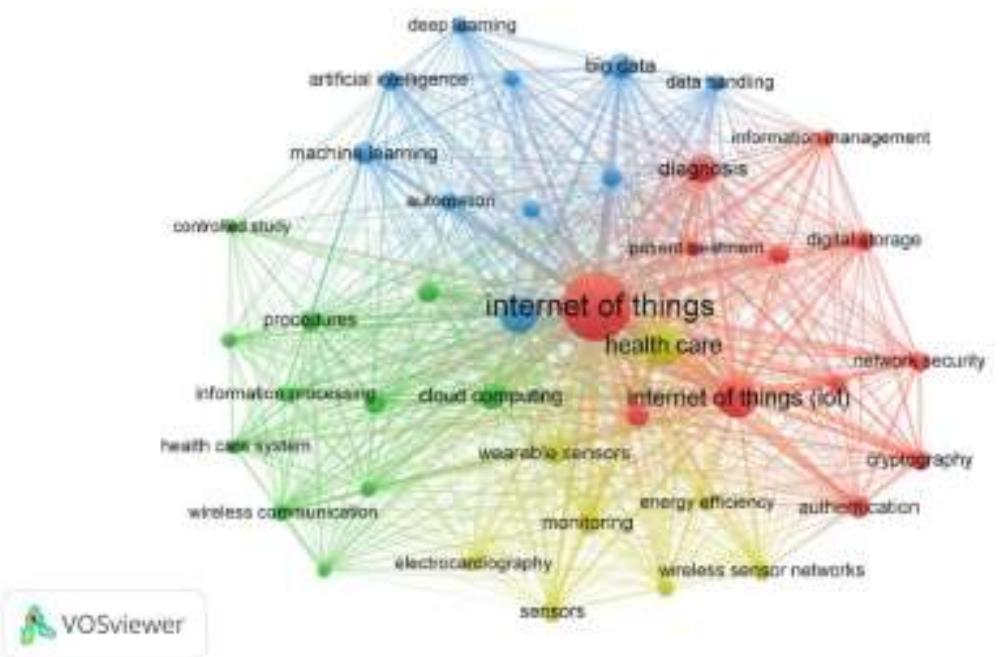

Fonte: Autores, a partir da base Scopus (2020). 
Pode-se notar ao menos quatro clusters principais, em que essas palavras possuem uma conexão mais forte umas com as outras. Além dos termos mais prováveis, é possível identificar diversas palavras relacionadas a tecnologias que compõe o paradigma da IoT, como por exemplo: wereable sensors, wireless communication, wireless sensor network. Outros termos estão relacionados a temas atuais da computação que também se relacionam com a IoT, como por exemplo: cloud computing, deep learning, machine learning, artificial inteligence e big data.

A big data é um tópico emergente da IoT, a demanda por suas análises integra a inovação mais recente em aprendizado de máquina, metodologias lógicas, inteligência computacional e mineração de dados. Especialistas em saúde agora estão analisando big data para obter conhecimento detalhado. Apesar de ainda estarem em fases iniciais, espera-se que ajudem coletivamente a resolver os problemas relacionados ao setor em relação à inconsistência na qualidade da saúde e ao aumento dos gastos com saúde (MORDOR INTELIGENCE, 2019). A robótica cirúrgica, que tem sido aliada à inteligência artificial, já está em uso em algum grau, mas poderá assumir um papel mais proeminente à medida que a tecnologia amadurece e faz uso da IoT. Sendo possível que esses robôs ultrapassem a precisão e capacidades dos cirurgiões humanos mais qualificados (DIGITAL INFORMATION WORLD, 2020).

\subsection{Patentes versus artigos}

Analisar um paralelo de evolução de depósito de patentes versus publicações de artigos pode ser importante para compreender a maturidade de determinadas tecnologias. Conforme pode ser observado na Figura 11, apesar da similaridade da curva, nota-se que em quase todo período o número de patentes depositadas se sobressai ao número de artigos publicados. 
Figura 11 - Comparativo do quantitativo de artigos publicados e famílias de patentes publicados por ano.

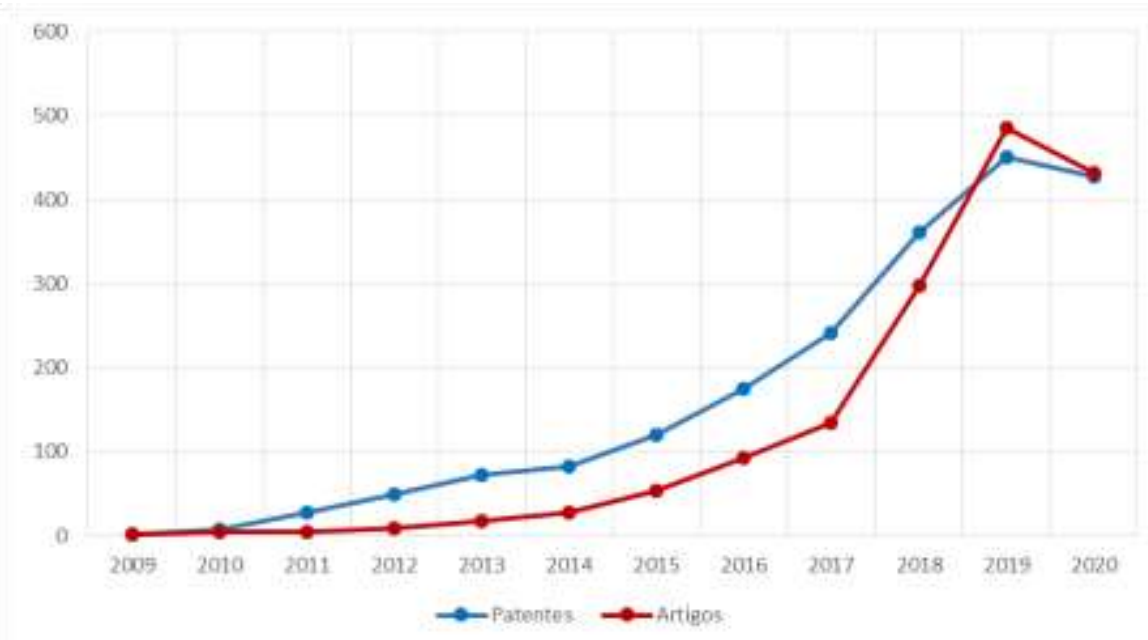

Fonte: O autor das bases Scopus e Questel-Orbit (2020).

Embora muitas tecnologias demandem anos de pesquisas para serem desenvolvidas, através dos investimentos em $\mathrm{P} \& \mathrm{D}$ por governos e pela própria iniciativa privada, nem sempre isso pode implicar em um grande número de publicações científicas. $\mathrm{O}$ foco no patenteamento de tecnologias pode ser explicado pela própria demanda de mercado, inclusive quando se trata de tecnologias computacionais, como é o caso da IoT, em que a velocidade em que essas tecnologias evoluem de um ano para outro, obrigam as empresas a protegerem rapidamente seus inventos, no intuito de terem vantagem competitiva frente a seus concorrentes.

\section{Considerações Finais}

Este estudo prospectivo apresentou a evolução dos pedidos de patentes e pesquisas científicas sobre IoT com aplicações no ambiente hospitalar no período de 2009 a 2020 . Foi possível notar um crescimento exponencial desses pedidos de patentes em especial a partir do ano de 2015, sendo o ano de 2019 o ano com maior número de patentes publicadas. O levantamento bibliométrico também apresentou considerável crescimento de publicações científicas sobre IoT no contexto de ambientes hospitalares, com um total de 1557 artigos, sendo o ano de 2019 o ano com o maior número de publicações.

Os resultados apontaram para a China, como uma forte região propulsora de tecnologias de IoT com aplicação em hospitais. Com um número de famílias de patentes bastante expressivo (1627), mais da metade do apurado neste estudo, a China supera os 
números de patentes de países como Índia, Coreia do Sul e Estados Unidos. Já a Índia, foi destacada como a principal nação em termos de publicações científicas sobre essa temática, com 441 artigos científicos publicados. Um dos fatores principais que colocam China e Índia, como nações que tem se interessado e investido cada vez mais nessa matéria, são suas políticas governamentais.

A análise da categorização das patentes levantadas por IPC, foi um importante indicador de tendências dessas tecnologias. As classificações de tecnologias de diagnóstico sendo especificamente sobre medições para fins de diagnóstico (A61B-005), e as tecnologias de transmissão de informações digitais, como disposições, aparelhos, circuitos ou sistemas (H04L-029), representam as classificações com os maiores números de famílias de patentes deste estudo. Já o IPC da família G16H, que tratam de informática da saúde, foram os que tiverem maior crescimento nesse período, em especial nos últimos quatro anos. Isso aponta para a forte tendência de digitalização do setor hospitalar com tecnologias que abrangem sistemas de telemonitoramento, tecnologias vestíveis e sensores corporais.

O panorama que apresentou uma clusterização do universo de patentes apurado apresentou uma alta concentração de patentes ligadas aos conceitos de intelligent diagnosis, platform management, real-time, home monitor, o que aponta para uma forte tendência em tecnologias inteligentes de monitoramento em tempo real e de diagnóstico. Dentre os principais temas que têm sido abordados em estudos científicos, foi possível observar temas emergentes da computação e que têm sido grandes aliados do paradigma da IoT, como: aprendizado de máquina, deep learning, big data, inteligência artificial e cloud computing.

De modo geral, é presumível que a pandemia do novo Coronavírus tenha sido um divisor de águas na área da assistência médica, o que poderá levar as nações a refletirem sobre a importância dessas tecnologias e seus benefícios que ainda não são explorados. Em tempos de monitoramento remoto de pacientes, controle de infecções hospitalares, teleconsultas, e diagnósticos rápido, é possível que o mercado da tecnologia da IoT hospitalar avance ainda mais nos próximos anos, aumentando a competitividade entre os principais players do mercado, e por outro lado modernizando as tradicionais instalações hospitalares.

Dentro do vasto ecossistema da saúde, uma infinidade de temas pode ser explorada em estudos futuros. O próprio ambiente hospitalar é passível de aplicação de diversas categorias de tecnologias, desde aplicações na gestão administrativa do hospital até as próprias aplicações de tratamentos médicos. Assim, devido à importância deste setor para sociedade, mercado e academia, alguns estudos são propostos para pesquisas futuras: 
a) Panorama tecnológico de prontuários eletrônicos inteligentes; b) Prospecção de tecnologias de IoT ligadas ao controle de infecções hospitalares; c) Monitoramento científico de tecnologias de IoT para o combate à COVID-19.

\section{Referências}

Alencar, M. S. M. (2008). Estudo de Futuro Através da Aplicação de Técnicas de Prospecção Tecnológica: o caso da nanotecnologia. Tese (Doutorado em Ciências) - Universidade Federal do Rio de Janeiro, Escola de Química, Rio de Janeiro.

Anixter. (2018). Global Technology Briefing - Smart Hospital Infrastructure Best Practices. Anixter Inc. Relatório. Recuperado de https://www.anixter.com/content/dam/anixter/resources/brochures/anixter-iaap-healthcarebest-practices-report-en.pdf .

Cabra, J., Castro, D., Colorado, J., Mendez, D., \& Trujillo, L. (2017). An IoT Approach for Wireless Sensor Networks Applied to e-Health Environmental Monitoring. 2017 IEEE International Conference on Internet of Things (iThings) and IEEE Green Computing and Communications (GreenCom) and IEEE Cyber, Physical and Social Computing (CPSCom) and IEEE Smart Data (SmartData). pp. 578-583. doi: 10.1109/iThings-GreenCom-CPSComSmartData.2017.91.

Colakovic, A., \& Hadzialic, M. (2018). Internet of Things (IoT): A review of enabling technologies, challenges, and open research issues. Computer Networks, 144, pp. 17-39. doi: https://doi.org/10.1016/j.comnet.2018.07.017.

Chen, B., Baur, A., Stepniak, M., \& Wang, J. (2019). Finding the future of care provision: The role of smart hospitals. McKinsey \& Company. Disponível em: https://www.mckinsey.com/industries/healthcare-systems-and-services/our-insights/findingthe-future-of-care-provision-the-role-of-smart-hospitals.

Chen, J., Walz, E., Lafferty, B., Mcreynolds, J., Green, K., Ray, J., \& Mulvenon, J. (2018). China's Internet of Things. U.S.-China Economic and Security Review Commission. Recuperado em: https://www.uscc.gov/research/chinas-internet-things . 
Chatterjee, S., \& Kar, A. K. (2018). Regulation and governance of the Internet of Things in India. Digital Policy, Regulation and Governance, 20(5), pp. 399-412. doi: https://doi.org/10.1108/DPRG-04-2018-0017.

Digital Information World. (2020). IoT in Healthcare Expectations for 2020. Recuperado de https://www.digitalinformationworld.com/2020/02/iot-in-healthcare-expectations-for2020.html\#.

Enisa. (2016). Smart Hospitals - Security and Resilience for Smart Health Service and Infrastructures. European Union Agency For Network And Information Security (ENISA). Recuperado de https://www.enisa.europa.eu/publications/cyber-security-and-resilience-forsmart-hospitals/at_download/fullReport.

Gartner. (2019). Hype Cycle for the Internet of Things. Recuperado de https://www.gartner.com/en/documents/3947474/hype-cycle-for-the-internet-of-things-2019.

Gonçalves, C. (2017). Como a Internet das Coisas está transformando o mercado de saúde. $\begin{array}{lllll}\text { Revista } & \text { Hospitais Brasil. } & \text { Ed } & \text { Recuperado de }\end{array}$ https://issuu.com/publimededitora/docs/rhb_87_site .

Gil, A. C.. (2002). Como Elaborar Projetos de Pesquisa. Editora Atlas. São Paulo, Ed. 4.

Healthcare Global. (2014) . What three companies are dominating China's healthcare market?. Recuperado em: https://www.healthcareglobal.com/technology-and-ai-3/what-threecompanies-are-dominating-chinas-healthcare-market/.

Healthcare Global. (2014). IoT: enabling next-gen healthcare services. Recuperado em: https://www.healthcareglobal.com/technology-and-ai-3/iot-enabling-next-gen-healthcareservices.

Ilin, I., Ilyaschenko, O., \& Korandi, A. (2018). Business model for Smart Hospital health organization. SHS of Conferences, $44 . \quad$ doi: https://doi.org/10.1051/shsconf/20184400041. 
IOT Analytics. (2020). Top 10 IoT applications in 2020. Recuperado em https://iotanalytics.com/top-10-iot-applications-in-2020/.

ISCOOP. (2020). Internet of Things (IoT) in healthcare: benefits, use cases and evolutions. Recuperado de https://www.i-scoop.eu/internet-of-things-guide/internet-things-healthcare/.

Lin, S., Wang, I. L., Lin, C., \& Chiueh, H. (2018). An Ultra-Low Power Smart Headband for Real-Time Epileptic Seizure Detection. IEEE J Transl Eng Health Med, 6. Doi: 10.1109/JTEHM.2018.2861882.

Liu, Z., Cao, Y., Cui, L., Song, J., \& Zhao, G. (2018). A Benchmark Database and Baseline Evaluation for Fall Detection Based on Wearable Sensors for the Internet of Medical Things Platform. IEEE Access, 6. pp. 51286-51296. doi: 10.1109/ACCESS.2018.2869833.

Kumar, S., Tiwari, P., \& Zymbler, M. (2019). Internet of Things is a revolutionary approach for future technology enhancement: a review. Journal of Big Data. 6(111). doi: https://doi.org/10.1186/s40537-019-0268-2.

Kajol, H., Junaid, S., Jain, S., Chaudhary, A., \& Prajwal, K. T. (2019). Design and Development of a Voice Actuated Hospital Bed for Patient Care. International Journal of Recent Technology and Engineering (IJRTE), 8(4), pp. 1751-1757. doi: 10.35940/ijrte.C5882.118419.

Kulkarni, A., \& Sathe, S. (2014). Healthcare applications of the Internet of Things: A Review. International Journal of Computer Science and Information Technologies, 5(5), pp. 62296232. Recuperado em: https://www.semanticscholar.org/paper/Healthcare-applications-ofthe-Internet-of-Things-\%3A-Kulkarni-Sathe/33822617ce5a200360d5871bea6e5a4cc88659b7

Magrani, E. (2018). A Internet das Coisas. Rio de Janeiro. Editora FGV. Recuperado em: https://bibliotecadigital.fgv.br/dspace/bitstream/handle/10438/23898/A\%20internet\%20das\% 20coisas.pdf. 
Majunder, Akm. J. A., Elsaadany, Y. A., Young Jr, R. \& Ucci, D. R. (2019). An Energy Efficient Wearable Smart IoT System to Predict Cardiac Arrest. Hindawi Advances in Human-Computer Interaction, 2019. doi: https://doi.org/10.1155/2019/1507465.

Mendes, C. U. S. (2012). Estudos de Monitoramento Tecnológico. INPI. Apresentação em slides. Recuperado de http://www.redemineirapi.com/novo/wp-content/uploads/I-dia.pdf.

Mobidev. (2020). 8 Technology Trends in Healthcare to Watch in 2020. Recuperado de https://mobidev.biz/blog/technology-trends-healthcare-digital-transformation.

Mordor Intelligence. (2019). Internet of Things (IoT) in Healthcare Market - Growth, Trends and Forecast (2020-2025). Recuperado de https://www.mordorintelligence.com/industryreports/internet-of-things-in-healthcare-market.

Orbit Intelligence. (2020). [Base de dados - Internet]. Questel Orbit Inc. Recuperado em: https://www.orbit.com/.

OMPI. (2014). Informação Tecnológica. Curso DL101P BR, Módulo 11. Organização Mundial de Propriedade Intelectual/Instituto Nacional da Propriedade Industrial. 2014.

Pires, P., Delicato, F., Batista, T. V., Avila, T., Cavalcante, E., \& Pitanga, M. (2015). Plataforma para Internet das Coisas. In: Simpósio Brasileiro de Rede de Computadores e Sistemas Distribuídos, n. 33․ Livro Texto Minicursos. Sociedade Brasileira de Computação, Porto Alegre, p.110-169. Recuperado de https://sbrc2015.ufes.br/wpcontent/uploads/Ch3.pdf.

Raj, S. P., Kavitha, N. S., Rachel, S. S., \& Suganthi, P.. (2019). Smart Hospitals E-Medico Management System. International Journal of Recent Technology and Engineering (IJRTE). 8(3). doi: : 10.35940/ijrte.C6841.098319.

Riad, K., Hamza, R., Yan, H. (2019). Sensitive and Energetic IoT Access Control for Managing Cloud Electronic Health Records. IEEE Access Special Section on Security and Privacy in Emerging Descentralized Communication Enviroments, 7. pp. 86384 - 86393. doi: 10.1109/ACCESS.2019.2926354. 
Scopus. (2020). [Base de dados - Internet]. Elsevier.. Recuperado de https://www.scopus.com/.

Silva, E. L., \& Menezes, E. M.. (2005). Metodologia da Pesquisa e Elaboração de Dissertação. Laboratório de Ensino a Distância da UFSC, ed. 4, Florianópolis.

Takhare, V.,\& Khire, G. (2014). Role of Emerging Technology for Building Smart Hospital Information System. Procedia Economics and Finance. 11(14), p.583-588. doi: https://doi.org/10.1016/S2212-5671(14)00223-8.

Yu, L., Lu, Y., \& Zhu, X. (2012). Smart Hospital based on Internet of Things. Journal of Networks, 7(10), p.1654-1661. doi: 10.4304/jnw.7.10.1654-1661.

Zheng, X., \& Rodriguez-Monroy, C. (2015). The Development of Intelligent Healthcare in China. Telemedicine and E-health. 21(5), pp. 443-448. doi: 10.1089/tmj.2014.0102.

\section{Porcentagem de contribuição de cada autor no manuscrito}

Felipe Roberto Eloi Moura - 50\%

Guilherme Benjamin Brandão Pitta - 30\%

Josealdo Tonholo - 20\% 